\title{
Hiccups, CTCAE
}

National Cancer Institute

\section{Source}

National Cancer Institute. Hiccups, CT CAE. NCI Thesaurus. Code C143545.

A disorder characterized by repeated gulp sounds that result from an involuntary opening and closing of the glottis. This is attributed to a spasm of the diaphragm. 